\title{
On the norm-preservation of squares in real algebra representation
}

\author{
F. Albiac ${ }^{1}$ (D) $\cdot$ O. Blasco ${ }^{2} \cdot$ E. Briem ${ }^{3}$ \\ Received: 27 April 2021 / Accepted: 10 July 2021 \\ (c) The Author(s) 2021, corrected publication 2021
}

\begin{abstract}
One of the main results of the article Gelfand theory for real Banach algebras, recently published in [Rev R Acad Cienc Exactas Fís Nat Ser A Mat RACSAM 114(4):163, 2020] is Proposition 4.1, which establishes that the norm inequality $\left\|a^{2}\right\| \leq\left\|a^{2}+b^{2}\right\|$ for $a, b \in \mathcal{A}$ is sufficient for a commutative real Banach algebra $\mathcal{A}$ with a unit to be isomorphic to the space $\mathcal{C}_{\mathbb{R}}(\mathcal{K})$ of continuous real-valued functions on a compact Hausdorff space $\mathcal{K}$. Moreover, in this proposition is also shown that if the above condition (which involves all the operations of the algebra) holds, then the real-algebra isomorphism given by the Gelfand transform preserves the norm of squares. A very natural question springing from the above-mentioned result is whether an isomorphism of $\mathcal{A}$ onto $\mathcal{C}_{\mathbb{R}}(\mathcal{K})$ is always norm-preserving of squares. This note is devoted to providing a negative answer to this problem. To that end, we construct algebra norms on spaces $\mathcal{C}_{\mathbb{R}}(\mathcal{K})$ which are $(1+\epsilon)$-equivalent to the sup-norm and with the norm of the identity function equal to 1 , where the norm of every nonconstant function is different from the standard sup-norm. We also provide examples of two-dimensional normed real algebras $\mathcal{A}$ where $\left\|a^{2}\right\| \leq k\left\|a^{2}+b^{2}\right\|$ for all $a, b \in \mathcal{A}$, for some $k>1$, but the inequality fails for $k=1$.
\end{abstract}

Keywords Real commutative Banach algebra $\cdot$ Real algebra homomorphism $\cdot \mathcal{C}(\mathcal{K})$-space Representation of algebras · Gelfand theory

Mathematics Subject Classification 46J10

F. Albiac

fernando.albiac@unavarra.es

O. Blasco

oscar.blasco@uv.es

E. Briem

briem@hi.is

1 Deparment of Mathematics, Statistics, and Computer Sciences and InaMat2, Universidad Pública de Navarra, Campus de Arrosadía, 31006 Pamplona, Spain

2 Department of Mathematical Analysis, Universidad de Valencia, 46100 Burjassot, Valencia, Spain

3 Science Institute University of Iceland, 107 Reykjavik, Iceland 


\section{Introduction}

The papers [2,3] show how certain very simple inequalities involving either the algebra norm or the spectral radius imply that a real commutative unital Banach algebra is homomorphic, via the Gelfand transform, to $\mathcal{C}_{\mathbb{R}}(\mathcal{K})$, the algebra of all continuous real-valued functions on a compact Hausdorff space $\mathcal{K}$ equipped with the usual norm,

$$
\|f\|_{\infty, \mathcal{K}}=\max _{x \in \mathcal{K}}|f(x)| .
$$

In [3, Theorem 1.1] it is shown that if $\mathcal{A}$ is a commutative real Banach algebra with unit, then the spectral radius $r$ satisfies the inequality

$$
r\left(a^{2}\right) \leq r\left(a^{2}+b^{2}\right), \text { for all } a, b \in \mathcal{A},
$$

if and only if the Gelfand transform

$$
\Lambda: \mathcal{A} \rightarrow \mathcal{C}_{\mathbb{C}}(\mathcal{K}), \quad a \hookrightarrow \widehat{a}
$$

maps into $\mathcal{C}_{\mathbb{R}}(\mathcal{K})$.

What happens if we just know that the spectral radius satisfies instead the (a priori weaker) inequality

$$
r\left(a^{2}\right) \leq k r\left(a^{2}+b^{2}\right), \text { for all } a, b \in \mathcal{A},
$$

for some $k \geq 1$ ? The answer is that nothing new happens. Indeed, the fulfilment of condition (1.2) for some $k \geq 1$ implies that the spectrum of any $a \in \mathcal{A}$ is a subset of the real line (see [2, Proposition 3.5]) and hence using [3, Theorem 1.2], we see that inequality (1.2) is satisfied with $k=1$.

In regards to isomorphisms we have the following.

Proposition 1.1 Let $\mathcal{A}$ be a commutative real algebra with unit. Suppose $\mathcal{A}$ is isomorphic to $\mathcal{C}_{\mathbb{R}}(\mathcal{K})$ for some compact Hausdorff space $\mathcal{K}$. Then the spectral radius seminorm $r$ on $\mathcal{A}$ is equivalent to the algebra norm (hence in particular $r$ defines a norm on $\mathcal{A}$ ). Moreover, $\mathcal{A}$ equipped with $r$ is isometric to $\mathcal{C}_{\mathbb{R}}(\mathcal{K})$.

Proof Let $\|\cdot\|$ denote the norm on $\mathcal{A}$. Suppose $\Lambda: \mathcal{A} \rightarrow \mathcal{C}_{\mathbb{R}}(\mathcal{K})$ is an isomorphism and let $k$ and $k_{1}$ be constants so that

$$
\frac{1}{k_{1}}\|a\| \leq\|\Lambda(a)\|_{\infty, \mathcal{K}} \leq k\|a\|, \quad a \in \mathcal{A}
$$

For $a$ in $\mathcal{A}$ we have

$$
\left\|a^{n}\right\| \leq k_{1}\left\|\Lambda\left(a^{n}\right)\right\|_{\infty, \mathcal{K}}=k_{1}\|\Lambda(a)\|_{\infty, \mathcal{K}}^{n} .
$$

Taking the $n$ th-root and letting $n$ tend to infinity yields $r(a) \leq\|\Lambda(a)\|_{\infty, \mathcal{K}}$.

Conversely,

$$
\|\Lambda(a)\|_{\infty, \mathcal{K}}^{n}=\left\|\Lambda\left(a^{n}\right)\right\|_{\infty, \mathcal{K}} \leq k\left\|a^{n}\right\| .
$$

Taking the $n$th root and letting $n$ tend to infinity yields $\|\Lambda(a)\|_{\infty, \mathcal{K}} \leq r(a)$, so that $\|\Lambda(a)\|_{\infty, \mathcal{K}}=r(a)$ as claimed.

In this paper we shall be concerned with normed real algebras satisfying the corresponding inequality (1.2), where the spectral radius is replaced by the algebra norm. Let us assign a tag to such a class of real algebras. 
Definition 1.2 Suppose $\mathcal{A}$ is a commutative real Banach algebra with unit and let $k \geq 1$. We will say that $\mathcal{A}$ satisfies property $(A)_{k}$, to be denoted $\mathcal{A} \in(A)_{k}$, if the following inequality holds

$$
\|a\|^{2} \leq k\left\|a^{2}+b^{2}\right\|, \quad a, b \in \mathcal{A} .
$$

In turn, we will say that $\mathcal{A}$ satisfies property $(B)_{k}$, to be denoted $\mathcal{A} \in(B)_{k}$, if

$$
\left\|a^{2}\right\| \leq k\left\|a^{2}+b^{2}\right\|, \quad a, b \in \mathcal{A} .
$$

Of course, $\mathcal{A} \in(A)_{k}$ implies $\mathcal{A} \in(B)_{k}$.

It was shown in [1, Proposition 3.3] that $\mathcal{A} \in \bigcup_{k \geq 1}(A)_{k}$ if and only if $\mathcal{A} \in \bigcup_{k \geq 1}(B)_{k}$. In [1, Theorem 3.6] the authors also proved that if $\mathcal{A} \in \bigcup_{k \geq 1}(B)_{k}$ then $\mathcal{A}$ is isomorphic to the algebra $\mathcal{C}_{\mathbb{R}}(\mathcal{K})$ for some compact Hausdorff space $\mathcal{K}$. The next example shows that $\mathcal{A}$ can be isomorphic to $\mathcal{C}_{\mathbb{R}}(\mathcal{K})$ and yet $\mathcal{A} \notin \bigcup_{k \geq 1}(B)_{k}$.

Example 1.3 Consider the algebra of matrices

$$
\mathcal{A}=\left\{a=\left(\begin{array}{ll}
x & y \\
0 & x
\end{array}\right): x, y \in \mathbb{R}\right\}
$$

endowed with the norm on each $a \in \mathcal{A}$ regarded as an operator on $\left(\mathbb{R}^{2},\|\cdot\|_{2}\right)$. Since $\mathcal{A}$ is two-dimensional, it is isomorphic to $\left(\mathbb{R}^{2},\|\cdot\|_{\infty}\right)$. The matrix $a=\left(\begin{array}{ll}0 & 1 \\ 0 & 0\end{array}\right)$ verifies $a^{2}=0$; however, there is no $k>0$ such that $\|a\|^{2} \leq k\left\|a^{2}\right\|$. This shows that $\mathcal{A} \notin \bigcup_{k \geq 1}(A)_{k}=$ $\bigcup_{k \geq 1}(B)_{k}$.

A natural question arises: Do we have a similar situation as with the spectral radius? i.e., does it hold that $\bigcup_{k \geq 1}(A)_{k}=(A)_{1}$ or $\bigcup_{k \geq 1}(B)_{k}=(B)_{1}$ ? The answer to this question for property $(A)_{k}$ is clearly negative. Recall that if $\mathcal{A} \in(A)_{1}$, i.e.,

$$
\|a\|^{2} \leq\left\|a^{2}+b^{2}\right\|, \quad a, b \in \mathcal{A},
$$

then $\mathcal{A}$ is isometrically isomorphic to the algebra $\mathcal{C}_{\mathbb{R}}(\mathcal{K})$ for some compact Hausdorff space $\mathcal{K}$ (see $[4,5]$ ). Hence it suffices to equip $\mathcal{C}_{\mathbb{R}}(\mathcal{K})$ with some equivalent algebra norm.

On the other hand, the condition that $\mathcal{A} \in(B)_{1}$, i.e.,

$$
\left\|a^{2}\right\| \leq\left\|a^{2}+b^{2}\right\|, \quad a, b \in \mathcal{A},
$$

only guarantees that $\mathcal{A}$ is isomorphic to the algebra $\mathcal{C}_{\mathbb{R}}(\mathcal{K})$ for some compact Hausdorff space $\mathcal{K}$, although in general it needs not be isometric. In the example where $\mathcal{A}=\mathcal{C}_{\mathbb{R}}(\mathcal{K})$ equipped with the algebra norm $\|f\|=\left\|f^{+}\right\|_{\infty, \mathcal{K}}+\left\|f^{-}\right\|_{\infty, \mathcal{K}}$ the condition (1.6) is satisfied but $\mathcal{A}$ is not isometric to any $\mathcal{C}_{\mathbb{R}}(\mathcal{K})$. However, we have the following extra information.

Proposition 1.4 (cf. [3, Proposition 4.1]) Let $\mathcal{A}$ be a commutative real Banach algebra with unit. Then $\mathcal{A} \in(B)_{1}$ if and only if there exists a compact Hausdorff space $\mathcal{K}$ and an $\mathbb{R}$-algebra isomorphism

$$
\Lambda: \mathcal{A} \rightarrow \mathcal{C}_{\mathbb{R}}(\mathcal{K}), \quad a \hookrightarrow \widehat{a},
$$

which preserves the norm of squares, i.e.,

$$
\left\|\widehat{a}^{2}\right\|_{\infty, \mathcal{K}}=\left\|a^{2}\right\|, \quad a \in \mathcal{A} .
$$


The question arises whether an $\mathbb{R}$-algebra isomorphism of $\mathcal{A}$ onto $\mathcal{C}_{\mathbb{R}}(\mathcal{K})$ is always normpreserving on squares.

As the alert reader might have guessed, if a commutative real Banach algebra with unit $\mathcal{A}$ is isomorphic to a space $\mathcal{C}_{\mathbb{R}}(\mathcal{K})$ for some compact Hausdorff space $\mathcal{K}$ then $\mathcal{K}$ must agree with the set $\Phi_{\mathcal{A}}^{\mathbb{R}}$ of all real homomorphisms of the algebra, and the isomorphism must be the Gelfand transform (see [2, Remark 2.8]). So the above question will be answered negatively, by constructing in Theorem 2.1 some algebra norm in $\mathcal{C}_{\mathbb{R}}(\mathcal{K})$ equivalent to $\|\cdot\|_{\infty, \mathcal{K}}$ which does not preserve the norm of squares.

Of course the above result also exhibits an example of a real normed algebra $\mathcal{A} \in$ $\bigcup_{k>1}(B)_{k} \backslash(B)_{1}$. Now, Theorem 2.5 will allow us to produce a number of such examples simply by considering two-dimensional normed algebras $\mathcal{A}$ such that there exists $v \in \mathcal{A}$ with $v^{2}=v$ and $\|v\|>1$. We will prove this in the following section.

For notation and background we refer the reader to the recent article [3], which this note aims to complement.

\section{Main theorems}

Theorem 2.1 Let $\mathcal{K}$ be a compact Hausdorff space with more than two points. For each $\epsilon>0$ we can construct a norm $\|\cdot\|_{\epsilon}$ on $\mathcal{C}_{\mathbb{R}}(\mathcal{K})$ with the following properties:

(i) For all $f$ in $\mathcal{C}_{\mathbb{R}}(\mathcal{K})$,

$$
\|f\|_{\infty, \mathcal{K}} \leq\|f\|_{\epsilon} \leq(1+2 \epsilon)\|f\|_{\infty, \mathcal{K}} .
$$

(ii) For all $f$ and $g$ in $\mathcal{C}_{\mathbb{R}}(\mathcal{K})$,

$$
\left\|f^{2}\right\|_{\epsilon} \leq(1+\epsilon)\left\|f^{2}+g^{2}\right\|_{\epsilon} .
$$

(iii) $\left\|f^{2}\right\|_{\epsilon}>\left\|f^{2}\right\|_{\infty, \mathcal{K}}$ for all functions $f \in \mathcal{C}_{\mathbb{R}}(\mathcal{K})$ such that $f^{2}$ is nonconstant.

Moreover, the constants $1+2 \epsilon$ in (2.1) and $1+\epsilon$ in (2.2) are sharp.

Proof For $f \in \mathcal{C}_{\mathbb{R}}(\mathcal{K})$ we define

$$
\|f\|_{\epsilon}=\|f\|_{\infty, \mathcal{K}}+\epsilon \sup _{k_{1} \neq k_{2}}\left|f\left(k_{1}\right)-f\left(k_{2}\right)\right| .
$$

It is clear that $\|\cdot\|_{\epsilon}$ is a norm on $\mathcal{C}_{\mathbb{R}}(\mathcal{K})$ that satisfies

$$
\|f\|_{\infty, \mathcal{K}} \leq\|f\|_{\epsilon} \leq(1+2 \epsilon)\|f\|_{\infty, \mathcal{K}}, \quad f \in \mathcal{C}_{\mathbb{R}}(\mathcal{K}) .
$$

Let us observe also that if $f, g \in \mathcal{C}_{\mathbb{R}}(\mathcal{K})$, and $k_{1}, k_{2} \in \mathcal{K}$ with $k_{1} \neq k_{2}$,

$$
\begin{aligned}
\left|f\left(k_{1}\right) g\left(k_{1}\right)-f\left(k_{2}\right) g\left(k_{2}\right)\right| & \leq\left|f\left(k_{1}\right)-f\left(k_{2}\right)\right|\left|g\left(k_{1}\right)\right|+\left|f\left(k_{2}\right)\right|\left|g\left(k_{1}\right)-g\left(k_{2}\right)\right| \\
& \leq\left|f\left(k_{1}\right)-f\left(k_{2}\right)\right|\|g\|_{\infty, \mathcal{K}}+\left|g\left(k_{1}\right)-g\left(k_{2}\right)\right|\|f\|_{\infty, \mathcal{K}} .
\end{aligned}
$$

Hence,

$$
\begin{aligned}
\|f g\|_{\epsilon}= & \|f g\|_{\infty, \mathcal{K}}+\epsilon \sup _{k_{1} \neq k_{2}}\left|f\left(k_{1}\right) g\left(k_{1}\right)-f\left(k_{2}\right) g\left(k_{2}\right)\right| \\
\leq & \|f\|_{\infty, \mathcal{K}}\|g\|_{\infty, \mathcal{K}}+\epsilon \sup _{k_{1} \neq k_{2}}\left|f\left(k_{1}\right)-f\left(k_{2}\right)\right|\|g\|_{\infty, \mathcal{K}} \\
& +\epsilon \sup _{k_{1} \neq k_{2}}\left|g\left(k_{1}\right)-g\left(k_{2}\right)\right|\|f\|_{\infty, \mathcal{K}} \leq\|f\|_{\epsilon}\|g\|_{\epsilon} .
\end{aligned}
$$


The constant $1+2 \epsilon$ in (i) is sharp since we can pick points $k_{1} \neq k_{2}$ in $\mathcal{K}$ and a function $f \in \mathcal{C}_{\mathbb{R}}(\mathcal{K})$ with $\|f\|_{\infty, \mathcal{K}}=1$ such that $f\left(k_{1}\right)=1$ and $f\left(k_{2}\right)=-1$. Therefore, $\|f\|_{\epsilon}=$ $1+2 \epsilon$.

Now observe that if $f \in \mathcal{C}_{\mathbb{R}}(\mathcal{K})$ with $0 \leq f(k) \leq 1$ then $\|f\|_{\epsilon} \leq 1+\epsilon$. Thus, if $\|f\|_{\infty, \mathcal{K}}=1$ and $g \in \mathcal{C}_{\mathbb{R}}(\mathcal{K})$,

$$
\left\|f^{2}\right\|_{\epsilon} \leq(1+\epsilon) \leq(1+\epsilon)\left\|f^{2}+g^{2}\right\|_{\infty, \mathcal{K}} \leq(1+\epsilon)\left\|f^{2}+g^{2}\right\|_{\epsilon} .
$$

To see that the constant $1+\epsilon$ in the last inequality is sharp, choose $f \in \mathcal{C}_{\mathbb{R}}(\mathcal{K})$ with $0 \leq f \leq 1$ taking the values 0 and 1 , and $g \in \mathcal{C}_{\mathbb{R}}(\mathcal{K})$ such that $f^{2}+g^{2}=1$.

Finally, note that if $f \in \mathcal{C}_{\mathbb{R}}(\mathcal{K})$ is such that $f\left(k_{1}\right) \neq f\left(k_{2}\right)$ for some $k_{1} \neq k_{2}$ in $\mathcal{K}$, then $\left\|f^{2}\right\|_{\epsilon}>\left\|f^{2}\right\|_{\infty, \mathcal{K}}$.

We now give some results concerning the class $(B)_{k}$ for $k \geq 1$.

Proposition 2.2 Suppose $\mathcal{A} \in(B)_{k}$ for some $k \geq 1$. Then the formula

$$
\|a\|=\sqrt{\left\|a^{2}\right\|}, \quad a \in \mathcal{A},
$$

defines a quasi-norm on $\mathcal{A}$ such that

$$
\|a\| \leq\|a\| \leq k(1+\sqrt{\|e\|})^{2}\|a\|, \quad a \in \mathcal{A} .
$$

Proof Of course, $\|\mid a\| \leq\|a\|$ and clearly $\||\lambda a|\|=|\lambda|\||\|\mid\|$ for all $\lambda \in \mathbb{R}$ and $a \in \mathcal{A}$. The triangle law of the quasi-norm follows easily as well:

$$
\begin{aligned}
\|a+b\|^{2} & =\left\|(a+b)^{2}\right\| \leq k\left\|(a+b)^{2}+(a-b)^{2}\right\| \\
& =2 k\left\|a^{2}+b^{2}\right\| \\
& \leq 2 k\left(\left\|a^{2}\right\|+\left\|b^{2}\right\|\right) \\
& \leq 2 k(\|a\|+\|b\|)^{2},
\end{aligned}
$$

so that $\||a+b \|| \leq \sqrt{2 k}(\|a\||+\|b \mid\|)$.

Let us now show that

$$
\|a\| \leq k(\|a\|+\sqrt{\|e\|})^{2}, \quad a \in \mathcal{A} .
$$

Indeed,

$$
\begin{aligned}
\|4 a\| & =\left\|(a+e)^{2}-(a-e)^{2}\right\| \\
& \leq\left\|(a+e)^{2}\right\|+\left\|(a-e)^{2}\right\| \\
& =\|a+e\|^{2}+\|\|-e \|^{2} \\
& \leq 4 k(\|a\|+\sqrt{\|e\|})^{2} .
\end{aligned}
$$

If we plug $t a$ in inequality (2.4) we obtain

$$
t\|a\| \leq k(t\|\| a \|+\sqrt{\|e\|})^{2} .
$$

Therefore $\|\mid a\|=0$ implies $\|a\| \leq \frac{k}{t}\|e\|$ for all $t>0$ and so $a=0$. This shows that $\|\cdot \mid\|$ is a quasi-norm. Finally, using homogeneity we also obtain from (2.4) that

$$
\|a\| \leq k(1+\sqrt{\|e\|})^{2}\|a\|, \quad a \in \mathcal{A} .
$$


Remark 2.3 Notice that if $\mathcal{A} \in \bigcup_{k \geq 1}(B)_{k}$ then there does not exist $a \in \mathcal{A} \backslash\{0\}$ with $a^{2}=0$.

Lemma 2.4 Let $\mathcal{A}$ be a commutative normed real algebra with unit e. The following are equivalent:

(i) There exists $u \in \mathcal{A} \backslash\{0, \pm e\}$ such that $u^{2}=e$.

(ii) There exists $v \in \mathcal{A} \backslash\{0, \pm e\}$ such that $v^{2}=v$.

Proof This follows readily since $u^{2}=e$ iff $v^{2}=v$ for $v=\frac{e+u}{2}$.

Theorem 2.5 Let $\mathcal{A}$ be a two-dimensional commutative real algebra with unit e of norm $\|e\|=1$. Assume that there exists $u \in \mathcal{A} \backslash\{ \pm e\}$ such $u^{2}=e$. Then $\mathcal{A} \in(B)_{\|u\|}$. Moreover, $\|u\|=1$ if and only if $\mathcal{A}$ is isometrically isomorphic to $\left(\mathbb{R}^{2},\|\cdot\|_{\infty}\right)$.

Proof Write $v=\frac{e+u}{2}$ and $w=\frac{e-u}{2}$. Hence $v-w=u, v+w=e, v^{2}=v, w^{2}=w$ and $v w=0$. For each $a \in \mathcal{A}$ we can write $a=\alpha v+\beta w$. Therefore $a v=\alpha v$ and $a w=\beta w$, which gives

$$
\max \{|\alpha|,|\beta|\} \leq\|a\| .
$$

On the other hand, since $a=\frac{\alpha+\beta}{2} e+\frac{\alpha-\beta}{2} u$ and $\|u\| \geq 1$ we obtain

$$
\|a\| \leq\|u\|\left(\frac{|\alpha+\beta|}{2}+\frac{|\alpha-\beta|}{2}\right) \leq\|u\| \max \{|\alpha|,|\beta|\} .
$$

Therefore

$$
\max \{|\alpha|,|\beta|\} \leq\|a\| \leq\|u\| \max \{|\alpha|,|\beta|\} .
$$

To show that $\mathcal{A} \in(B)_{\|u\|}$ just notice that if $a=\alpha v+\beta w$ then $a^{2}=\alpha^{2} v+\beta^{2} w$. Hence, if $b=\alpha^{\prime} v+\beta^{\prime} w$ then $a^{2}+b^{2}=\left(\alpha^{2}+\left(\alpha^{\prime}\right)^{2}\right) v+\left(\beta^{2}+\left(\beta^{\prime}\right)^{2}\right) w$ and we can write

$$
\begin{aligned}
\left\|a^{2}\right\| & \leq\|u\| \max \left\{\alpha^{2}, \beta^{2}\right\} \\
& \leq\|u\| \max \left\{\alpha^{2}+\left(\alpha^{\prime}\right)^{2}, \beta^{2}+\left(\beta^{\prime}\right)^{2}\right\} \\
& \leq\|u\|\left\|a^{2}+b^{2}\right\| .
\end{aligned}
$$

Using also (2.5) we obtain that $\|u\|=1$ if and only if $\|a\|=\max \{|\alpha|,|\beta|\}$.

Corollary 2.6 Let $\mathcal{A}$ be a two-dimensional commutative real algebra with unit e of norm $\|e\|=1$. Assume that there exists $v \in \mathcal{A}$ such that $v^{2}=v$ and $\|v\|>1$. Then $\mathcal{A} \in$ $\bigcup_{k>1}(B)_{k} \backslash(B)_{1}$.

Proof Taking $u=2 v-e$ in Theorem 2.5 we have that $\mathcal{A} \in(B)_{\|2 v-e\|}$. To show that $\mathcal{A} \notin(B)_{1}$, if suffices to plug $a=v$ and $b=e-v$ in (1.4), since $\left\|a^{2}\right\|=\|v\|>1=\left\|a^{2}+b^{2}\right\|$.

Example 2.7 Let $\mathcal{A}$ be the algebra of all real-valued functions on a set $\mathcal{K}$ of two elements. Let $e$ denote the constant function 1 and $u$ denote a function which takes the value 1 at one of the points of $\mathcal{K}$ and -1 at the other. These two functions form a basis for $\mathcal{A}$. Let $\lambda>0$ and for $f=x_{1} e+x_{2} u$ define

$$
\|f\|_{\lambda, 1}=\left|x_{1}\right|+(1+\lambda)\left|x_{2}\right| .
$$

If $f$ takes the values $\alpha$ and $\beta$ then $x_{1}=\frac{\alpha+\beta}{2}$ and $x_{2}=\frac{\alpha-\beta}{2}$. Hence $\left|x_{1}\right|+\left|x_{2}\right|=\max \{|\alpha|,|\beta|\}$. Thus the above expression is just the norm in Theorem 2.1 (with $\mathcal{K}$ having only two points) for $\varepsilon=\lambda / 2$.

Using Theorem 2.5 and Corollary 2.6 with $v=\frac{u+e}{2}$ we infer that $\left(\mathcal{A},\|\cdot\|_{\lambda, 1}\right) \in(B)_{1+\lambda} \backslash$ $(B)_{1}$ since $\|u\|_{\lambda, 1}=1+\lambda$ and so $\|v\|_{\lambda, 1}=1+\frac{\lambda}{2}>1$. 
Let us now present some examples of norms on two-dimensional commutative real algebras with a unit satisfying the properties in Corollary 2.6. The construction is inspired by examples of two-dimensional Hilbert space operator algebras from [6].

Example 2.8 Let $\mathcal{A}$ be the set of matrices

$$
\left\{a=\left(\begin{array}{ll}
x_{1} & x_{2} \\
x_{2} & x_{1}
\end{array}\right): x_{1}, x_{2} \in \mathbb{R}\right\}
$$

with the usual matrix multiplication. For each $\lambda>0$ define

$$
\|a\|_{\lambda}=\sqrt{x_{1}^{2}+x_{2}^{2}}+\lambda\left|x_{2}\right|, \quad a=\left(\begin{array}{ll}
x_{1} & x_{2} \\
x_{2} & x_{1}
\end{array}\right) \in \mathcal{A} .
$$

It is easy to check that $\|\cdot\|_{\lambda}$ is a norm.

Theorem 2.9 Let $\mathcal{A}_{\lambda}=\left(\mathcal{A},\|\cdot\|_{\lambda}\right)$. Then $\mathcal{A}_{\lambda}$ is a normed algebra (i.e., $\|\cdot\|_{\lambda}$ is submultiplicative) if and only if $\lambda \geq \sqrt{2}$. Moreover, $\mathcal{A}_{\lambda} \in(B)_{1+\lambda} \backslash(B)_{1}$ for all $\lambda \geq \sqrt{2}$.

Proof Assume first that $\lambda \geq \sqrt{2}$. Set

$$
a=\left(\begin{array}{ll}
x_{1} & x_{2} \\
x_{2} & x_{1}
\end{array}\right), \quad b=\left(\begin{array}{ll}
y_{1} & y_{2} \\
y_{2} & y_{1}
\end{array}\right) .
$$

We need to show that $\|a b\|_{\lambda} \leq\|a\|_{\lambda}\|b\|_{\lambda}$. The case $x_{2} y_{2}=0$ follows trivially since either $a=x_{1} e$ or $b=y_{1} e$ where $e=\left(\begin{array}{ll}1 & 0 \\ 0 & 1\end{array}\right)$ and $\|a\|=\left|x_{1}\right|$ or $\|b\|=\left|y_{1}\right|$. We may assume that $x_{2} \neq 0$ and $y_{2} \neq 0$, so that it suffices to check the above inequality for

$$
a=\left(\begin{array}{ll}
x & 1 \\
1 & x
\end{array}\right), \quad b=\left(\begin{array}{ll}
y & 1 \\
1 & y
\end{array}\right)
$$

where $x, y \in \mathbb{R} \backslash\{0\}$. Thus we need to show that

$$
\sqrt{(x y+1)^{2}+(x+y)^{2}}+\lambda|x+y| \leq\left(\sqrt{1+x^{2}}+\lambda\right)\left(\sqrt{1+y^{2}}+\lambda\right),
$$

or, equivalently,

$$
\sqrt{\left(1+x^{2}\right)\left(1+y^{2}\right)+4 x y}-\sqrt{\left(1+x^{2}\right)\left(1+y^{2}\right)} \leq \lambda\left(\sqrt{1+x^{2}}+\sqrt{1+y^{2}}-|x+y|+\lambda\right) .
$$

Since

$$
\sqrt{1+x^{2}}+\sqrt{1+y^{2}}-|x+y|>0
$$

it suffices to see that

$$
\frac{4 x y}{\sqrt{\left(1+x^{2}\right)\left(1+y^{2}\right)+4 x y}+\sqrt{\left(1+x^{2}\right)\left(1+y^{2}\right)}} \leq \lambda^{2} .
$$

We may assume that $x y>0$. Observe that

$$
\frac{4 x y}{\sqrt{\left(1+x^{2}\right)\left(1+y^{2}\right)+4 x y}+\sqrt{\left(1+x^{2}\right)\left(1+y^{2}\right)}} \leq \frac{2 x y}{\sqrt{\left(1+x^{2}\right)\left(1+y^{2}\right)}} \leq 2,
$$

which gives (2.6) for all $\lambda \geq \sqrt{2}$. 
Assume now that $\mathcal{A}_{\lambda}$ is a normed algebra. In particular for $a_{t}=\left(\begin{array}{ll}t & 1 \\ 1 & t\end{array}\right)$ we have $\left\|a_{t}^{2}\right\|_{\lambda} \leq$ $\left\|a_{t}\right\|_{\lambda}^{2}$ for all $t>0$. Since

$$
a_{t}^{2}=\left(\begin{array}{cc}
t^{2}+1 & 2 t \\
2 t & t^{2}+1
\end{array}\right)
$$

we infer that

$$
\sqrt{\left(1+t^{2}\right)^{2}+4 t^{2}} \leq\left(\sqrt{t^{2}+1}+\lambda\right)^{2}-2 t \lambda \leq t^{2}+1+\lambda^{2}+2 \lambda\left(\sqrt{1+t^{2}}-t\right) .
$$

Therefore,

$$
\sqrt{\left(1+t^{2}\right)^{2}+4 t^{2}}-\left(1+t^{2}\right)=\frac{4 t^{2}}{\sqrt{\left(1+t^{2}\right)^{2}+4 t^{2}}+\left(1+t^{2}\right)} \leq \lambda^{2}+\frac{2 \lambda}{\sqrt{1+t^{2}}+t} .
$$

Taking limits as $t \rightarrow \infty$ gives $\lambda^{2} \geq 2$.

Finally, use that $u=\left(\begin{array}{ll}0 & 1 \\ 1 & 0\end{array}\right)$ satisfies $u^{2}=e$ and $\|u\|_{\lambda}=1+\lambda$, and that $v=\left(\begin{array}{ll}1 / 2 & 1 / 2 \\ 1 / 2 & 1 / 2\end{array}\right)$ satisfies $v^{2}=v$ and $\|v\|=\frac{\sqrt{2}}{2}+\frac{\lambda}{2} \geq \sqrt{2}$ and invoke Theorem 2.5 and Corollary 2.6 to see that $\mathcal{A}_{\lambda} \in(B)_{1+\lambda} \backslash(B)_{1}$.

We can set the above examples in a general scale.

Example 2.10 Let $\mathcal{A}=\mathbb{R}^{2}$ and set $e=(1,1)$ and $u=(1,-1)$. Given $a=x_{1} e+x_{2} u$ and $b=y_{1} e+y_{2} u$ we have

$$
a b=\left(x_{1} y_{1}+x_{2} y_{2}\right) e+\left(x_{1} y_{2}+x_{2} y_{1}\right) u .
$$

Let $\lambda>0$ and $1 \leq p \leq \infty$. For $a=x_{1} e+x_{2} u$ we define

$$
\|a\|_{\lambda, p}=\left\|\left(x_{1}, x_{2}\right)\right\|_{p}+\lambda\left|x_{2}\right|,
$$

where, as usual, $\left\|\left(x_{1}, x_{2}\right)\right\|_{p}=\left(\left|x_{1}\right|^{p}+\left|x_{2}\right|^{p}\right)^{1 / p}$ for $1 \leq p<\infty$, and $\left\|\left(x_{1}, x_{2}\right)\right\|_{\infty}=$ $\max \left\{\left|x_{1}\right|,\left|x_{2}\right|\right\}$

Using Examples 2.7 and 2.8 we can enunciate the following result about the normed algebra $\mathcal{A}=\left(\mathbb{R}^{2},\|\cdot\|_{\lambda, p}\right)$.

Proposition 2.11 Let $\mathcal{A}$ be $\mathbb{R}^{2}$ equipped with the norm $\|\cdot\|_{\lambda, p}$ defined in (2.7).

(i) If $p=1$ then $\mathcal{A} \in(B)_{1+\lambda} \backslash(B)_{1}$ for all $\lambda>0$.

(ii) If $p=2$ then $\|\cdot\|_{\lambda, 2}$ is submultiplicative for all $\lambda \geq \sqrt{2}$. Moreover, $\mathcal{A} \in(B)_{1+\lambda} \backslash(B)_{1}$ for all $\lambda \geq \sqrt{2}$.

Proposition 2.12 Let $\lambda>0$. Then $\|\cdot\|_{\lambda, \infty}$ is a submultiplicative norm on $\mathbb{R}^{2}$ if and only if $\lambda \geq 1$. Moreover, if $\mathcal{A}$ is $\mathbb{R}^{2}$ equipped with the norm $\|\cdot\|_{\lambda, \infty}$, then $\mathcal{A} \in(B)_{1+\lambda} \backslash(B)_{1}$ for $\lambda>1$.

Proof Assume that $\|\cdot\|_{\lambda, \infty}$ is submultiplicative. If $a=e+u$ we have

$$
\left\|a^{2}\right\|_{\lambda, \infty}=2\|a\|_{\lambda, \infty} \leq\|a\|_{\lambda, \infty}^{2} .
$$

Hence $1+\lambda=\|a\|_{\lambda, \infty} \geq 2$ and so $\lambda \geq 1$. 
Assume now that $\lambda \geq 1$. We only need to analyze the cases $a=x e+u$ and $b=y e+u$. We have

$$
\begin{aligned}
\|a\|_{\lambda, \infty} & =\max \{|x|, 1\}+\lambda, \\
\|b\|_{\lambda, \infty} & =\max \{|y|, 1\}+\lambda, \text { and } \\
\|a b\|_{\lambda, \infty} & =\max \{|x y+1|,|x+y|\}+\lambda|x+y| .
\end{aligned}
$$

We want $\|a b\|_{\lambda, \infty} \leq\|a\|_{\lambda, \infty}\|b\|_{\lambda, \infty}$, i.e.,

$$
\max \{|x y+1|,|x+y|\}+\lambda|x+y| \leq(\max \{|x|, 1\}+\lambda)(\max \{|y|, 1\}+\lambda) .
$$

Let us show that

$$
\max \{|x||y|+1,|x|+|y|\}+\lambda(|x|+|y|) \leq(\max \{|x|, 1\}+\lambda)(\max \{|y|, 1\}+\lambda) .
$$

When $|x| \leq 1$ and $|y| \leq 1$ the right hand-side is equal to $(1+\lambda)^{2}$ and the left hand-side is at most $2+2 \lambda$, so the inequality holds.

Assuming $|x| \geq 1$ and $|y| \geq 1$ the inequality becomes

$$
(|x||y|+1)+\lambda(|x|+|y|) \leq(|x|+\lambda)(|y|+\lambda),
$$

which also holds for $\lambda \geq 1$.

In the case when $|x| \leq 1 \leq|y|$ the inequality becomes

$$
(1+\lambda)(|x|+|y|) \leq(1+\lambda)(|y|+\lambda),
$$

which follows again under the assumption of $\lambda \geq 1$.

To finish the proof we just need to apply Theorem 2.5 and Corollary 2.6 with $v=\frac{e+u}{2}$.

Proposition 2.13 Let $\lambda>0$ and $1<p<2$. Then the norm $\|\cdot\|_{\lambda, p}$ is not submultiplicative.

Proof Assume that $\|\cdot\|_{\lambda, p}$ is submultiplicative. Then $\left\|a^{2}\right\|_{\lambda, p} \leq\|a\|_{\lambda, p}^{2}$ for $a=t e+u$ and $t>0$. Therefore,

$$
\left(\left(t^{2}+1\right)^{p}+(2 t)^{p}\right)^{1 / p}-\left(t^{p}+1\right)^{2 / p} \leq \lambda\left(2\left(t^{p}+1\right)^{1 / p}-2 t+\lambda\right) .
$$

Observe now that $\phi_{p}(t)=\left(t^{p}+1\right)^{1 / p}-t$ is decreasing and $0 \leq \phi_{p}(t) \leq 1$. Hence the right hand-side of (2.8) is bounded by $\lambda^{2}+2 \lambda$. On the other hand,

$$
\begin{aligned}
& \lim _{t \rightarrow \infty}\left(\left(t^{2}+1\right)^{p}+(2 t)^{p}\right)^{1 / p}-\left(t^{p}+1\right)^{2 / p} \\
& =\lim _{s \rightarrow 0} \frac{\left(\left(s^{2}+1\right)^{p}+(2 s)^{p}\right)^{1 / p}-\left(s^{p}+1\right)^{2 / p}}{s^{2}} \\
& =\lim _{s \rightarrow 0} \frac{\left(\left(s^{2}+1\right)^{p}+(2 s)^{p}\right)^{1 / p-1}\left(s^{2-p}\left(1+s^{2}\right)^{p-1}+2^{p-1}\right)-\left(1+s^{p}\right)^{2 / p-1}}{s^{2-p}} \\
& =\infty .
\end{aligned}
$$

This gives a contradiction, and so there is no $\lambda>0$ for which $\|\cdot\|_{\lambda, p}$ is submultiplicative.

To analyze the case $\|\cdot\|_{\lambda, p}$ for $p>2$ we shall use the following lemma.

Lemma 2.14 Suppose $p>2$. For $(x, y) \in \mathbb{R}^{2}$ put

$$
\Phi_{p}(x, y)=\left((x y+1)^{p}+(x+y)^{p}\right)^{1 / p}-\left(x^{p}+1\right)^{1 / p}\left(y^{p}+1\right)^{1 / p} .
$$


Then if $x, y>0$,

$$
\Phi_{p}(x, y) \leq\left(1+\frac{1}{x y}\right)^{p-1} \min \{1, x y\}+2^{p-1}(\min \{x, y\})^{2-p} .
$$

In particular

$$
\Phi_{p}(x, y) \leq 2^{p}, \quad x, y>0 .
$$

Proof We will use the following elementary inequalities, where $p^{\prime}$ denotes the conjugate exponent of $p$, determined by the relation $1 / p+1 / p^{\prime}=1$ :

$$
\begin{aligned}
a^{1 / p}-b^{1 / p} \leq \frac{a-b}{p b^{1 / p^{\prime}}}, & a>b>0 \\
u^{p}-v^{p} \leq p u^{p-1}(u-v), & u>v>0
\end{aligned}
$$

and

$$
\alpha+\beta-\left(\alpha^{p}+\beta^{p}\right)^{1 / p} \leq \min \{\alpha, \beta\}, \quad \alpha, \beta>0 .
$$

Note that

$$
x y+1 \geq\left(x^{p} y^{p}+1\right)^{1 / p}
$$

and

$$
x+y \geq\left(x^{p}+y^{p}\right)^{1 / p},
$$

so that

$$
(x y+1)^{p}+(x+y)^{p} \geq\left(1+x^{p}\right)\left(1+y^{p}\right) .
$$

Hence, applying consecutively (2.11), (2.12), and (2.13) gives

$$
\begin{aligned}
\Phi_{p}(x, y) \leq & \frac{(x y+1)^{p}-\left(x^{p} y^{p}+1\right)+(x+y)^{p}-\left(x^{p}+y^{p}\right)}{p\left(1+y^{p}\right)^{1 / p^{\prime}}\left(1+x^{p}\right)^{1 / p^{\prime}}} \\
\leq & \frac{1}{(x y)^{p / p^{\prime}}}\left((x y+1)^{p-1}\left(x y+1-\left(x^{p} y^{p}+1\right)^{1 / p}\right)\right) \\
& +\frac{1}{(x y)^{p / p^{\prime}}}\left((x+y)^{p-1}\left(x+y-\left(x^{p}+y^{p}\right)^{1 / p}\right)\right) \\
\leq & \left(1+\frac{1}{x y}\right)^{p-1} \min \{1, x y\}+\left(\frac{1}{x}+\frac{1}{y}\right)^{p-1} \min \{x, y\} \\
\leq & \left(1+\frac{1}{x y}\right)^{p-1} \min \{1, x y\}+\left(\frac{2}{\min \{x, y\}}\right)^{p-1} \min \{x, y\} .
\end{aligned}
$$

Assume now that $x, y \geq 1$. Then, (2.9) and the condition $p>2$ give $\Phi_{p}(x, y) \leq 2^{p-1}+$ $2^{p-1}=2^{p}$ for all $x, y \geq 1$. But since $\Phi_{p}(x, y)=\Phi_{p}(y, x)$ and $\Phi_{p}(x, y)=x \Phi_{p}\left(\frac{1}{x}, y\right)$ for all $x, y>0$, we obtain that $\Phi_{p}(x, y) \leq 2^{p}$ for all $x, y>0$.

Proposition 2.15 For $2<p<\infty$ set

$$
A_{p}=\sup _{x>0, y>0} \Phi_{p}(x, y) .
$$

(i) If $\|\cdot\|_{\lambda, p}$ is submultiplicative then $\lambda \geq \frac{A_{p}}{\sqrt{1+A_{p}}+1}$. 
(ii) If $\lambda \geq 2^{p / 2}$ then $\|\cdot\|_{\lambda, p}$ is a submultiplicative norm on $\mathbb{R}^{2}$. In particular, if we consider $\mathcal{A}=\mathbb{R}^{2}$ equipped with this norm then $\mathcal{A} \in(B)_{1+\lambda} \backslash(B)_{1}$ for all $\lambda \geq 2^{p / 2}$.

Proof (i) Assume that $\|a b\|_{\lambda, p} \leq\|a\|_{\lambda, p}\|b\|_{\lambda, p}$ for $a=x e+u$ and $b=y e+u$ with $x, y>0$. Then,

$$
\begin{aligned}
\left((x y+1)^{p}+(x+y)^{p}\right)^{1 / p}- & \left(x^{p}+1\right)^{1 / p}\left(y^{p}+1\right)^{1 / p} \leq \\
& \lambda\left(\left(x^{p}+1\right)^{1 / p}-x+\left(y^{p}+1\right)^{1 / p}-y\right)+\lambda^{2} .
\end{aligned}
$$

In particular, using that $\left(x^{p}+1\right)^{1 / p}-x \leq 1$, we obtain

$$
\Phi_{p}(x, y) \leq \lambda^{2}+2 \lambda, \quad x, y>0 .
$$

This gives that $\lambda^{2}+2 \lambda-A_{p} \geq 0$ so that $\lambda \geq \sqrt{A_{p}+1}-1$.

(ii) Assume that $\lambda^{2} \geq 2^{p}$. Let $a=x_{1} e+y_{1} u$ and $b=x_{2} e+y_{2} u$. If $y_{1} y_{2}=0$ then $\|a b\|_{\lambda, p}=\|a\|_{\lambda, p}\|b\|_{\lambda, p}$. Hence we only need to check that $\|a b\|_{\lambda, p} \leq\|a\|_{\lambda, p}\|b\|_{\lambda, p}$ for $a=x e+u$ and $b=y e+u$ with $x, y>0$. Since $a b=(x y+1) e+(x+y) u$, we must equivalently show that

$$
\Phi_{p}(x, y) \leq \lambda\left(\left(x^{p}+1\right)^{1 / p}-x+\left(y^{p}+1\right)^{1 / p}-y+\lambda\right),
$$

which follows from (2.10).

We conclude the proof by applying again Theorem 2.5 with $\|u\|_{\lambda, p}=1+\lambda$ and Corollary 2.6 with $v=\frac{e+u}{2}$, since

$$
\|v\|_{\lambda, p}=\frac{2^{1 / p}+\lambda}{2} \geq \frac{2^{1 / p}+2^{p / 2}}{2}>1
$$

Remark 2.16 We now define algebra norms on $\mathcal{C}_{\mathbb{R}}(\mathcal{K})$ where $\mathcal{K}$ has more than two points. Let $p>2$ and $\lambda \geq 2^{p / 2}$.

For an element $f$ of $\mathcal{C}_{\mathbb{R}}(\mathcal{K})$ put

$$
\|f\|_{\lambda, p}=\sup _{F}\left\|\left.f\right|_{F}\right\|_{\lambda, p},
$$

where the supremum is taken over all two-point subsets $F$ of $\mathcal{K}$ and $\left\|\left.f\right|_{F}\right\|_{\lambda, p}$ is defined using the construction of Example 2.7 and the definition in Example 2.10. In this definition there are two possibilities for the function $u$, but both possibilities give the same norm because one is minus the other.

Let us see that the supremum is actually obtained. Consider the function

$$
F_{p, \lambda}(s, t)=\left(\left|\frac{f(s)+f(t)}{2}\right|^{p}+\left|\frac{f(s)-f(t)}{2}\right|^{p}\right)^{1 / p}+\left|\frac{f(s)-f(t)}{2}\right| \lambda,
$$

which is continuous on $\mathcal{K} \times \mathcal{K}$.

If $f \in \mathcal{C}_{\mathbb{R}}(\mathcal{K})$ is nonconstant and $\lambda>1$, there exists $(s, t) \in \mathcal{K} \times \mathcal{K}$ with $s \neq t$ such that

$$
F_{p, \lambda}(s, t) \geq\left|\frac{f(s)+f(t)}{2}\right|+\left|\frac{f(s)-f(t)}{2}\right| \lambda>\max \{|f(t)|,|f(s)|\} .
$$

Hence $\|F\|_{\infty}>\|f\|_{\infty}$.

The function $F_{p, \lambda}$ attains its largest value at a point $\left(s_{0}, t_{0}\right)$ with $s_{0} \neq t_{0}$. Set $F_{0}=\left\{s_{0}, t_{0}\right\}$. Then the supremum in (2.15) is attained at $\left\|\left.f\right|_{F_{0}}\right\|_{\lambda, p}$.

The results shown previously extend to these infinite dimensional Banach algebras. 
Acknowledgements The first two authors acknowledge the support from the Spanish Ministry for Science, Innovation, and Universities under Grant PGC2018-095366-B-I00 for Análisis Vectorial, Multilineal y Aproximación. F. Albiac was also supported by the Spanish Ministry for Science and Innovation under Grant PID2019-107701GB-I00 for Operators, lattices, and structure of Banach spaces.

Funding Open Access funding provided thanks to the CRUE-CSIC agreement with Springer Nature.

Open Access This article is licensed under a Creative Commons Attribution 4.0 International License, which permits use, sharing, adaptation, distribution and reproduction in any medium or format, as long as you give appropriate credit to the original author(s) and the source, provide a link to the Creative Commons licence, and indicate if changes were made. The images or other third party material in this article are included in the article's Creative Commons licence, unless indicated otherwise in a credit line to the material. If material is not included in the article's Creative Commons licence and your intended use is not permitted by statutory regulation or exceeds the permitted use, you will need to obtain permission directly from the copyright holder. To view a copy of this licence, visit http://creativecommons.org/licenses/by/4.0/.

\section{References}

1. Albiac, F., Briem, E.: Representations of real Banach algebras. J. Aust. Math. Soc. 88(3), 289-300 (2010)

2. Albiac, F., Briem, E.: Real Banach algebras as $\mathcal{C}(\mathcal{K})$ algebras. Q. J. Math. 63(3), 513-524 (2012)

3. Albiac, F., Briem, E.: Gelfand theory for real Banach algebras. Rev. R. Acad. Cienc. Exactas Fís Nat. Ser. A Mat. RACSAM 114(4), 163 (2020)

4. Albiac, F., Kalton, N.: A characterization of real $C(K)$ spaces. Am. Math. Monthly 111, 737-743 (2007)

5. Arens, R.: Representation of *-algebras. Duke Math. J. 14, 269-282 (1947)

6. Nakazi, T.: Two-dimensional Q-algebras. Linear Algebra Appl. 315, 197-205 (2000)

Publisher's Note Springer Nature remains neutral with regard to jurisdictional claims in published maps and institutional affiliations. 